\title{
Effects of Placental Transfusion by Delayed Cord Clamping or Umbilical Cord Milking versus Immediate Cord Clamping on Neonatal Outcomes in Very Low Birth Weight Neonates
}

\author{
Swosti Joshi', Mopelola Akintorin ${ }^{2}$, Alison Wiles ${ }^{3}$, Annamarie Arias ${ }^{2}$, \\ Louis Fogg ${ }^{4}$, Tuan Nguyen ${ }^{5^{*}}$ \\ ${ }^{1}$ Division of Neonatology, John H Stroger Hospital of Cook County, Chicago, IL, USA \\ ${ }^{2}$ Department of Pediatrics, John H Stroger Hospital of Cook County, Chicago, IL, USA \\ ${ }^{3}$ Chicago Medical School at Rosalind Franklin University of Medical Sciences, Chicago, IL, USA \\ ${ }^{4}$ Division of Statistics, Rush University Medical Center, Chicago, IL, USA \\ ${ }^{5}$ Department of Obstetrics and Gynecology, John H Stroger Hospital of Cook County, Chicago, IL, USA \\ Email: *tnguyen22002@yahoo.com
}

How to cite this paper: Joshi, S., Akintorin, M., Wiles, A., Arias, A., Fogg, L. and Nguyen, T. (2018) Effects of Placental Transfusion by Delayed Cord Clamping or Umbilical Cord Milking versus Immediate Cord Clamping on Neonatal Outcomes in Very Low Birth Weight Neonates. Open Journal of Obstetrics and Gynecology, 8, 1032-1039.

https://doi.org/10.4236/ojog.2018.811104

Received: August 31, 2018

Accepted: September 17, 2018

Published: September 20, 2018

Copyright ( 2018 by authors and Scientific Research Publishing Inc. This work is licensed under the Creative Commons Attribution International License (CC BY 4.0).

http://creativecommons.org/licenses/by/4.0/ (c) (i) Open Access

\begin{abstract}
Background: Delayed cord clamping (DCC) and umbilical cord milking (UCM) are two recently advocated interventions to enhance placental transfusion at birth. DCC and MCM might increase blood volume and help neonates to transition from fetal to neonatal circulation. Objective: To evaluate the effects of delayed cord clamping (DCC), umbilical cord milking (UCM), and immediate cord clamping (ICC) on very low birth weight (VLBW) neonates. Methods: At our institution, ICC was routinely done until December, 2012 when it was replaced by DCC. UCM was implemented in March, 2015. Neonatal outcomes were compared among ICC, DCC, and UCM. Results: A total of 94 neonates met inclusion criteria. Comparing with ICC neonates, DCC and UCM neonates had higher hematocrits, fewer blood transfusions, and lower incidence of chronic lung disease at 36 completed weeks of gestation. All groups were similar in APGAR scores, vasopressor use, intraventricular hemorrhage (IVH) and necrotizing enterocolitis (NEC). Conclusion: Compared to ICC, DCC and UCM appear to be more beneficial to VLBW neonates without apparent adverse risks.
\end{abstract}

\section{Keywords}

Delayed Cord Clamping, Umbilical Cord Milking, Very Low Birth Weight, Placental Transfusion 


\section{Introduction}

Current recommendations by the American College of Obstetrician and Gynecologists (ACOG) encourage the use of delayed umbilical cord clamping (DCC) in all premature neonates as a means of placental transfusion because of its significant associated benefits [1]. Despite substantial evidence that placental transfusion by delayed cord clamping improves transitional circulation, decreases the need for blood transfusion, and lowers the incidence of necrotizing enterocolitis (NEC) and intraventricular hemorrhage (IVH) in preterm neonates, there appears to be reluctance to adopt DCC. This may be due to concern about delayed neonatal resuscitation until the cord is clamped and cut [2]. To avoid this delay, the study of two distinct approaches to immediate resuscitation efforts that preserve placental transfusion was examined. One such effort is the use of the mobile trolley to initiate resuscitation at the mother's bedside. Mobile trolley resuscitation has been shown to be a safe and successful way to delay cord clamping in applicable settings [3] but significant planning and resources must be devoted to the installation and use of a trolley. Alternatively, the stripping of blood from the umbilical cord, or umbilical cord milking (UCM) as a means of placental transfusion has been hypothesized as a fast and effective way to provide the benefits of placental transfusion to the neonate [4].

Umbilical cord milking is a method of achieving increased placental transfusion, similar to delayed cord clamping, but with the advantages of a more rapid time frame. DCC requires at least a 30 - 60 second delay in cord clamping and cutting, whereas umbilical cord milking is an active process of pushing the blood from the placenta in the direction of the neonate that takes between 10 to 15 seconds. The shortened time frame of placental transfusion by UCM is appealing in situations where immediate umbilical cord clamping should be considered such as maternal hemodynamic instability, abnormal placentation, or when the neonate needs immediate resuscitation.

If UCM is shown to be similarly beneficial to DCC in preterm neonates, the burden of delaying resuscitation efforts is removed and the practice of placental transfusion methods may be more routinely adopted, even in cases for which immediate resuscitation is needed. Debate persists regarding the efficacy of DCC versus UCM, especially in regard to our very premature neonates. More studies are needed to evaluate the potential benefits and risks of umbilical cord milking in very low birth weight (VLBW) premature neonates.

\section{Objective}

Our study aimed to assess the effects of DCC and UCM as compared with ICC on VLBW neonates.

\section{Methods}

This study was conducted at a single tertiary care center (John H. Stroger County Hospital) in Chicago, Illinois and was approved by the institutional review board (IRB). 
Immediate cord clamping (ICC) was done routinely before December 2012 at our institution. Data on historical controls born between January 2009 and December 2012 by ICC were collected via chart review. In January 2013, delayed cord clamping (30 seconds) was standard practice per ACOG guidelines at our institution. Data on historical controls born between December 2012 and March 2015 by DCC were also collected via chart review. Umbilical cord milking became an option for standard practice in March of 2015 after IRB approval. Data were collected prospectively on neonates born between March 2015 and December 2016 by UCM. Both UCM and DCC are currently accepted practice and considered minimally risky; therefore, the type of intervention was left to the discretion of the obstetricians.

Entry criteria were a gestational age of less than 32 weeks or a birth weight of less than 1500 grams. Exclusion criteria included placenta previa or abruption, monochorionic twin gestation, $\mathrm{Rh}$ sensitization, congenital anomalies, and the obstetricians declining to perform the intervention secondary to being unaware of the study protocol. Only neonates who had a documented delay in cord clamping of at least 30 seconds and no more than 60 seconds were included. Neonates that received a delay in cord clamping for 60 seconds or more were excluded from the study

Labor and delivery staff attending the delivery recorded the time of delivery and the time of umbilical cord clamping. DCC was done by holding the neonate below the level of the placenta and waiting for at least 30 seconds before clamping the cord. UCM was done by holding the neonate at least $10 \mathrm{~cm}$ below the level of the placenta and milking at least $10 \mathrm{~cm}$ of the length of the cord towards the neonate over a 1 - 2 second duration. Then the cord was released and allowed to refill with blood for a 1 - 2 second pause between each milking motion. This was repeated twice for a total of at least three milking motions before the cord was clamped and cut.

Delivery room data including APGAR scores at one and five minutes, intubation in the delivery room, and admission temperature were recorded at the time of birth. Neonatal outcomes including hemoglobin at birth, transfusion in the first 72 hours of life, patent ductus arteriosus (PDA) requiring treatment, oxygen therapy at 36 weeks, intraventricular hemorrhage (IVH), retinopathy of prematurity (ROP), ventilator days and length of stay were assessed upon discharge of the neonate by chart review.

\section{Statistical Analysis}

Neonatal and obstetrical data were collected. Qualitative variables were expressed as frequency (percentage). Normality of distribution was checked graphically. Quantitative variables were expressed in term of mean \pm SD for normally distributed data. Median and range were used for non-Gaussian distribution. Microsoft Excel and SPSS 16.0 (Statistical Package for Social Sciences) software package were used for analysis. Analysis of variance (ANOVA) was done to 
analyze three groups. Post hoc t-test was done to compare difference between UCM and DCC groups and UCM and ICC groups. Student t-test was used to analyze differences in normally distributed data. Mann-Whitney test was used to analyze differences in data with non-Gaussian distribution. Chi square test and Fischer exact test were used to analyze categorical data.

\section{Results}

A total of 150 neonates was eligible for inclusion in the study born between January 2009 and December 2016. Previously mentioned exclusion criteria were applied as illustrated in Figure 1, resulting in a total of 94 neonates eligible for data analysis.

The baseline characteristics of the three groups (ICC, DCC, and UCM) are shown in Table 1. Despite the inclusion criterion of 32 weeks' gestational age, the birth weight restriction narrowed the population included in the study to a mean gestational age of $26.6 \pm 1.9$ weeks. The mean birth weight was $911 \pm 262$ grams. The incidence of chorioamnionitis was statistically significantly less in number in the DCC group than either the ICC group or the UCM group ( $\mathrm{p}=$ 0.02 ). Since all participants of the study were inborn, all received at least one dose of antenatal steroids. More neonates were born vaginally in the DCC group than either the ICC group or the UCM group $(p=0.001)$.

The delivery room data of the patients are described in Table 2. There were no statistically significant differences between the three groups in admission temperature, intubation rates in the delivery room, or one and five minute APGAR scores.

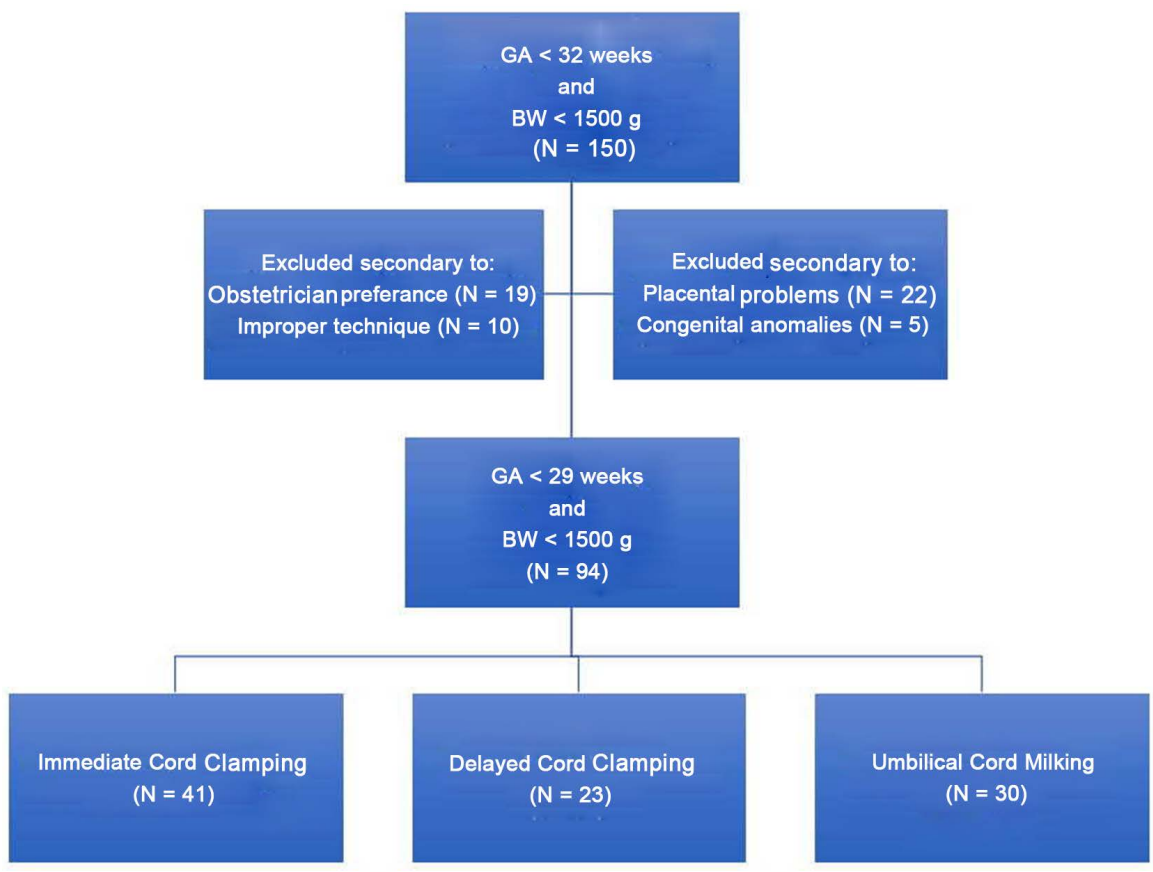

Figure 1. Enrollment of study participants specified by inclusion and exclusion criteria. 
Table 1. Baseline characteristics of groups.

\begin{tabular}{ccccc}
\hline & UCM $(\mathrm{N}=30)$ & DCC $(\mathrm{N}=23)$ & ICC $(\mathrm{N}=41)$ & p-value \\
\hline Maternal age, years & $26.2 \pm 5.1$ & $25.3 \pm 5.0$ & $25.9 \pm 6.6$ & 0.85 \\
Gestational age, weeks & $26.4 \pm 1.8$ & $26.4 \pm 1.9$ & $25.9 \pm 1.8$ & 0.43 \\
Birth weight, g & $890 \pm 283$ & $934 \pm 237$ & $909 \pm 266$ & 0.84 \\
$\quad$ Female & $18(60)$ & $8(35)$ & $21(51)$ & 0.18 \\
Chorioamnionitis & $3(10)$ & $0(0)$ & $1(2.5)$ & 0.02 \\
Any antenatal steroids & $30(100)$ & $22(95)$ & $41(100)$ & 0.21 \\
Caesarean section & $19(63)$ & $3(13)$ & $23(56)$ & 0.001 \\
\hline
\end{tabular}

Number in parenthesis $=$ percentage .

Table 2. Delivery room data of neonates.

\begin{tabular}{lcccc}
\hline & $\mathrm{UCM}(\mathrm{N}=30)$ & $\mathrm{DCC}(\mathrm{N}=23)$ & ICC $(\mathrm{N}=41)$ & p-value \\
\hline Admission temperature ${ }^{\circ} \mathrm{C}$ & $37.7 \pm 0.33$ & $36.9 \pm 0.44$ & $37.8 \pm 0.17$ & 0.45 \\
Intubation in delivery room & $29(96)$ & $17(74)$ & $33(80)$ & 0.62 \\
APGAR score in 1 minute & 6 & 7 & 5 & 0.14 \\
APGAR score in 5 minutes & 7 & 7.5 & 7 & 0.56 \\
\hline
\end{tabular}

Number in parenthesis $=$ percentage .

Neonatal outcomes of this study are shown in Table 3. Analysis revealed a statistically significant higher hemoglobin in the DCC and UCM groups in comparison with the ICC group $(\mathrm{p}=0.001)$. There was no significant difference in hemoglobin values between the DCC group and UCM group (mean $16.1 \mathrm{~g} / \mathrm{dL}$ and $15.0 \mathrm{~g} / \mathrm{dL}$ respectively). Data showed a statistically significantly lower rate of transfusion in the first 72 hours of life $(p=0.04)$ and a decreased need for oxygen at 36 weeks corrected $(p=0.04)$ in the DCC and UCM groups compared to the ICC group. There were fewer patients in the DCC and UCM group that had a PDA requiring treatment than in the ICC group, but this difference did not reach statistical significance $(p=0.07)$. The incidences of interventricular hemorrhage, retinopathy of prematurity, ventilator days and length of stay were similar in all three groups.

\section{Discussion}

Our study assessed the effects of DCC and UCM on VLBW neonates with the use of various cord clamping techniques. The study was initially designed to evaluate the effects of these cord clamping techniques on neonates of less than 32 weeks' gestation; however, birth weight exclusion criterion gives a better assessment of VLBW neonates of less than 29 weeks. Our data showed UCM was comparable to DCC in achieving higher hemoglobin status of the neonate at birth, and both were superior to ICC in this regard. The higher concentration of hemoglobin in neonates receiving some form of placental transfusion is a possible 
Table 3. Neonatal outcomes.

\begin{tabular}{ccccc}
\hline & UCM $(\mathrm{N}=30)$ & DCC $(\mathrm{N}=23)$ & ICC $(\mathrm{N}=41)$ & p-value \\
\hline Birth hemoglobin $(\mathrm{g} / \mathrm{dl})$ & $15.0 \pm 2.7$ & $16.1 \pm 2.9$ & $13.6 \pm 1.9$ & 0.001 \\
Transfusion < 72 hours of life & $9(30)$ & $3(13)$ & $18(44)$ & 0.04 \\
PDA requiring treatment & $6(20)$ & $1(4.3)$ & $12(30)$ & 0.07 \\
Oxygen at 36 weeks, corrected & $7(23)$ & $8(35)$ & $22(53)$ & 0.04 \\
Any IVH & $5(17)$ & $7(30)$ & $3(7.3)$ & 0.71 \\
Retinopathy of prematurity & $15(50)$ & $13(56)$ & $14(34)$ & 0.47 \\
Ventilator days & $11.3 \pm 19.1$ & $9.4 \pm 12.2$ & $10.5 \pm 15.0$ & 0.91 \\
Length of stay, days & $66.3 \pm 35.3$ & $74.04 \pm 35.9$ & $66.7 \pm 56.7$ & 0.96 \\
\hline
\end{tabular}

Number in parenthesis = percentage.

explanation for the reduced rate of blood transfusion in the first 72 hours of life in both the UCM and DCC groups when compared to the ICC group. This is important because transfusion of packed RBCs is under scrutiny in the neonatal literature due to concern that there is an association between packed RBC transfusion and morbidities such as necrotizing enterocolitis [5].

We also demonstrated a decreased need for oxygen at 36 weeks in both the UCM and DCC groups when compared to the ICC group. The incidence of bronchopulmonary dysplasia at 28 days of life was similar in all three groups, but the incidence of chronic lung disease (CLD) at 36 weeks of corrected gestational age was lower in UCM and DCC group than in ICC group. Improvement in CLD rates may be due to use of gentle ventilation over time, or the fact that the ICC group were historic controls. This is unlikely to be the case because the number of ventilation days in all three groups was not statistically significant. One possible explanation for the decrease in CLD rates in the placental transfusion groups could be that cord blood contributes to the improvement in long term oxygen need because of an increased transfer of stem cells in the neonatal circulation. Cord blood is rich in immunoglobulins and stem cells, which have potential for improved organ repair and rebuilding after injury caused by preterm birth [6].

Our data did not show UCM to be superior to DCC in any of the measures we assessed. A study by Katheria et al. [7] showed that neonates with UCM, who were born via caesarian section had higher superior venacava flow and right ventricular output than those with DCC. Such a difference was not seen during vaginal delivery. Katheria hypothesized that due to the uterine contractions at vaginal delivery, DCC may be sufficient to transfer all the available blood to the neonate. UCM may be particularly helpful in the cases where labor had not started and mother had undergone caesarean Section [8]. Due to lack of adequate uterine contractions, DCC may not be effective means of placental transfusion in those neonates. In our study, more neonates in the DCC group were born via vaginal delivery and more neonates in UCM were born via caesarean section. Route of delivery was a potentially confounding factor in our study. 
Additional weaknesses of our study include the relatively small sample size secondary to the low prevalence of VLBW neonates and inadequate data collection resulting in initial inclusion of a number of VLBW neonates that did not qualify for final statistical analysis.

It has been established in current literature that DCC is preferable to ICC in preterm neonates because of the numerous benefits including improved transitional circulation, decreases in the need for blood transfusion, and lower incidence of serious neonatal complications. Many physicians are reluctant to adopt the practice of DCC because of concern over the delay for neonate resuscitation efforts. UCM is being examined as a safe and effective means to provide placental transfusion while minimizing delay to resuscitation. Our study provides support to the hypothesis that VLBW neonates are able to derive similar benefits from UCM as they are from DCC. Therefore, UCM is useful in situations that require rapid cord clamping in order to initiate resuscitative measures. Multicenter studies are needed to confirm the results of this study in the population of VLBW neonates.

\section{Conclusion}

Our study demonstrates comparable benefits and no apparent increased risks in the use of DCC and UCM in VLBW neonates. The use of either method to enhance placental transfusion appears to be superior to ICC and may be considered as an option in the delivery of VLBW neonates to optimize outcomes.

\section{Conflicts of Interest}

The authors declare no conflict of interest.

\section{References}

[1] Committee on Obstetric Practice, American College of Obstetricians and Gynecologists (2012) Committee Opinion No. 543: Timing of Umbilical Cord Clamping after Birth. Obstetrics \& Gynecology, 120, 1522-1526. https://doi.org/10.1097/00006250-201212000-00045

[2] Ononeze, A.B. and Hutchon, D.J. (2009) Attitude of Obstetricians towards Delayed Cord Clamping: A Questionnaire Based Study. Journal of Obstetrics and Gynaecology, 29, 223-224. https://doi.org/10.1080/01443610802712918

[3] Thomas, M.R., Yoxall, C.W., Weeks, A.D. and Duley, L. (2014) Providing Newborn Resuscitation at the Mother's Bedside: Assessing the Safety, Usability and Acceptability of a Mobile Trolley. BMC Pediatrics, 14, 135 https://doi.org/10.1186/1471-2431-14-135

[4] Al-Wassia, H. and Shah, P.S. (2015) Efficacy and Safety of Umbilical Cord Milking at Birth: A Systemic Review and Meta-Analysis. JAMA Pediatrics, 169, 18-25. https://doi.org/10.1001/jamapediatrics.2014.1906

[5] Cunningham, K.E., Okolo, F.C., Baker, R., Mollen, K.O. and Good, M. (2017) Red Blood Cell Transfusion in Premature Infants Leads to Worse Necrotizing Enterocolitis Outcomes. Journal of Surgical Research, 213, 158-165. https://doi.org/10.1016/j.jss.2017.02.029 
[6] O'Reilly, M. (2013) The Promise of Stem Cells in Bronchopulmonary Dysplasia. Seminars in Perinatology, 37, 79-84. https://doi.org/10.1053/j.semperi.2013.01.003

[7] Katheria, A.C., Leone, T.A., Woelkers, D., Garey, D.M., Rich, W. and Finer, N.N. (2017) The Effects of Umbilical Cord Milking on Hemodynamics and Neonatal Outcomes in Premature Neonates. Journal of Pediatrics, 164, 1045-1050. https://doi.org/10.1016/j.jpeds.2014.01.024

[8] Katheria, A.C., Truong, G., Cousins, L., Oshiro, B. and Finer, N.N. (2015) Umbilical Cord Milking versus Delayed Cord Clamping in Premature Infants. Pediatrics, 136, 61-69. 